\title{
A Complete Postversion of the Three-Body Continuum Distorted Wave-Eikonal Initial State Approximation for Single Ionization of Multielectron Atoms
}

\author{
J. M. Monti, ${ }^{1}$ O. A. Fojón, ${ }^{1}$ J. Hanssen, ${ }^{2}$ and R. D. Rivarola ${ }^{1}$ \\ ${ }^{1}$ Instituto de Física Rosario (CONICET-UNR) and Facultad de Ciencias Exactas, Ingeniería y Agrimensura, \\ Universidad Nacional de Rosario, Avenida Pellegrini 250, 2000 Rosario, Argentina \\ ${ }^{2}$ Institut de Physique, Laboratoire de Physique Moléculaire et des Collisions, Université Paul Verlaine-Metz, \\ 1 Bv. Arago, $57078 \mathrm{Metz}$ Cedex 3, France \\ Correspondence should be addressed to J. M. Monti, monti@ifir-conicet.gov.ar
}

Received 14 December 2009; Accepted 25 February 2010

Academic Editor: Boris A. Malomed

Copyright (๑) 2010 J. M. Monti et al. This is an open access article distributed under the Creative Commons Attribution License, which permits unrestricted use, distribution, and reproduction in any medium, provided the original work is properly cited.

Single electron ionization of helium targets produced by the impact of fast proton beams is investigated. The postversion of the continuum distorted wave-eikonal initial state into a three-body approximation is reformulated, including the dynamic screening produced by the nonionized electron. This dynamic screening is shown to play a main role in the determination of double differential cross-sections. A good agreement is found with predictions obtained employing the prior version of the model, so that post-prior discrepancies are almost eliminated.

\section{Introduction}

Electron ionization in ion-atom collisions has been a subject of main interest in the last decades [1]. In order to accelerate the convergence of perturbative Born series, distorted wave models were introduced, as for example the continuum distorted wave [2] and the continuum distorted wave-eikonal initial state (CDW-EIS; [3]) ones. They were formulated for the case of monoelectronic targets, being the first-orders of the corresponding distorted wave series. Later, an extension for the case of multielectronic atoms was given by Fainstein et al. [4] reducing the multielectronic target case to a monoelectronic one.

The CDW-EIS model was applied with success to describe experiments for numerous collisional systems, where multiple and single differential as well as total ionization cross-sections were measured $[1,5]$. For multielectronic targets, the initial bound state was described by means of a Hartree-Fock wavefunction distorted by a multiplicative eikonal phase representing the active electron in a continuum state of the projectile field. In the final channel, the continuum of this active electron (the ionized one) was described by a double product of a plane wave and two continuum factors, each one of them associated with the residual target and the projectile fields. In this model, the passive electrons (those not ionized) are considered to remain as frozen in their initial orbitals. To facilitate the calculation of transition matrix elements and the corresponding cross sections, effective coulomb potentials were chosen to represent the interaction between the passive electrons and the active one in the exit channel. So, the active electron was considered as travelling in the combined fields of an effective coulomb continuum of the residual target and of the projectile. Thus, prior and postversions of the transition matrix elements gave place to different cross sections values. This was observed as a resulting post-prior discrepancy between these two versions $[6,7]$.

Furthermore, numerical initial bound and final continuum wavefunctions, corresponding to the same HartreeFock potential in both the entry and exit channels, respectively, were also considered to describe the active electronresidual target interaction in the CDW-EIS model [8]. The final continuum eigenstate was represented by a partialwave expansion, and the initial and final radial functions 


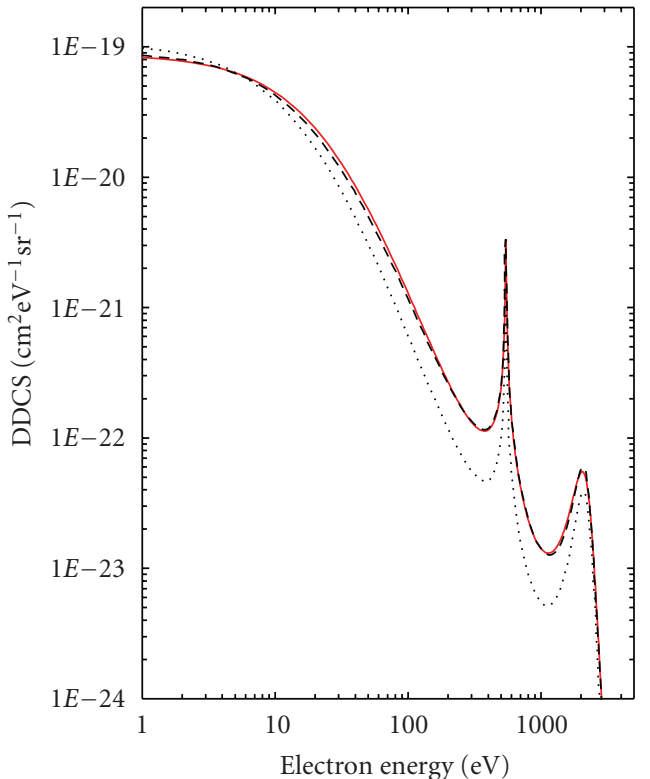

(a)

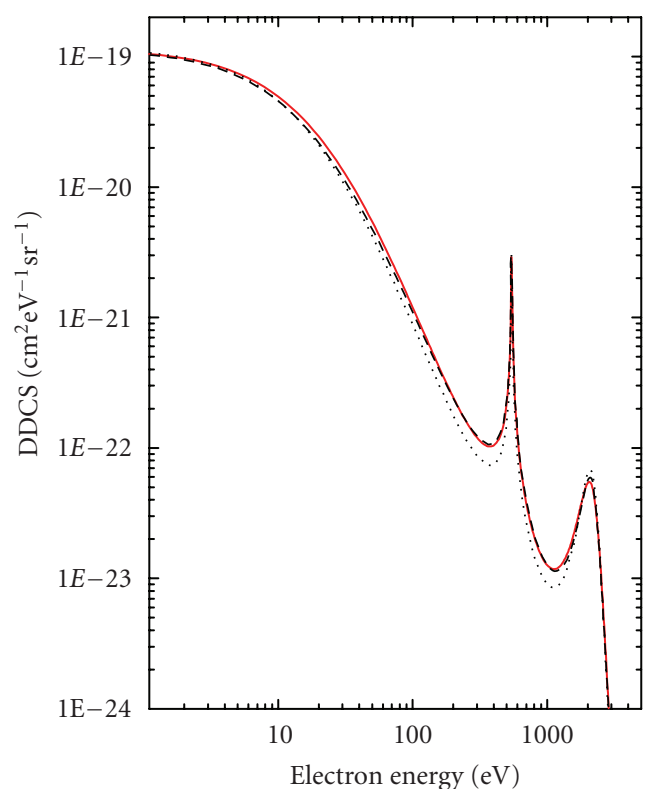

(b)

Figure 1: Doubly differential cross section for electron emission in collisions of $1 \mathrm{MeV} \mathrm{H}^{+}$on He targets as a function of electron energy for $0^{\circ}$ fixed ejection angle, considered in the residual target continuum function an asymptotic charge (a) or an effective charge (b). Theory: prior CDW-EIS; ..., post-CDW-EIS; - - , post-CDW-EIS including the terms $V_{T}^{\text {as }}$ (a) and $V_{T}^{\text {eff }}(\mathrm{b})$.

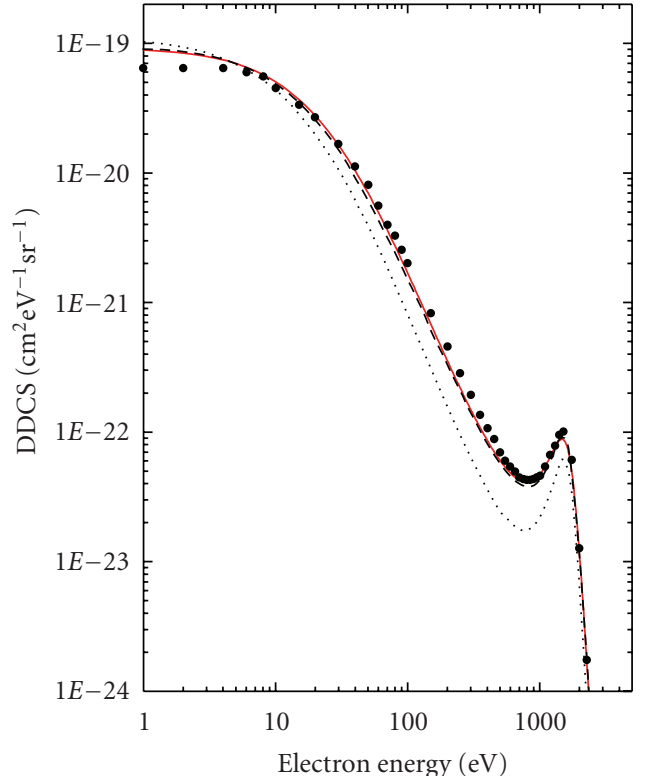

(a)

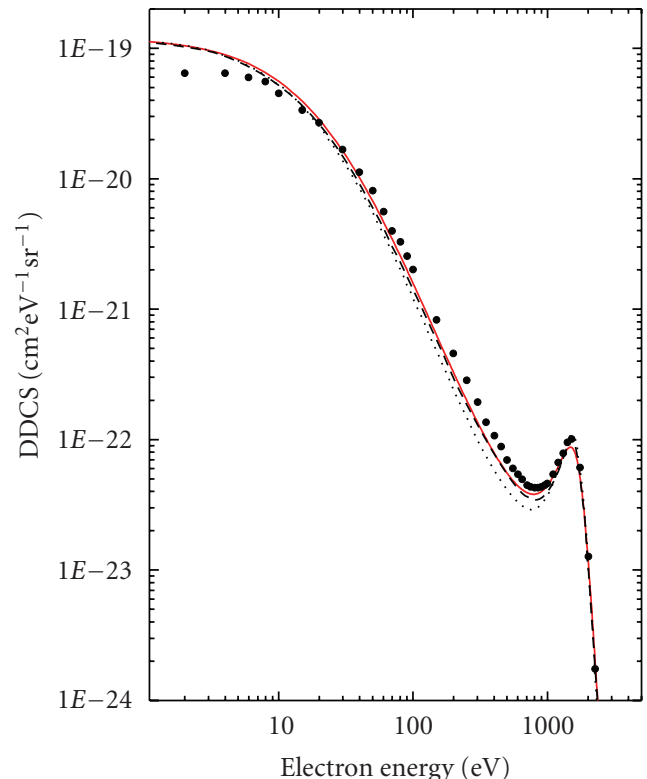

(b)

Figure 2: The same as Figure 1 but for $30^{\circ}$ fixed ejection angle. Experiment: •, extracted from Rudd et al. [12].

were determined by numerical integration of the radial Schrödinger equation with the Numerov algorithm. This choice for the wavefunctions avoids any post-prior discrepancy. However, their use involves a hard numerical computation of cross sections (see [8] for details).

In the present work we revisit the formulation of the post-version of the CDW-EIS approximation showing that a residual potential that was neglected in previous works must be included to obtain agreement with prior version calculations. This potential is associated with a correct description of the dynamic screening produced by the passive electrons on the evolution of the active one and its inclusion allows evaluation of the influence of this interaction on single ionization cross sections. 


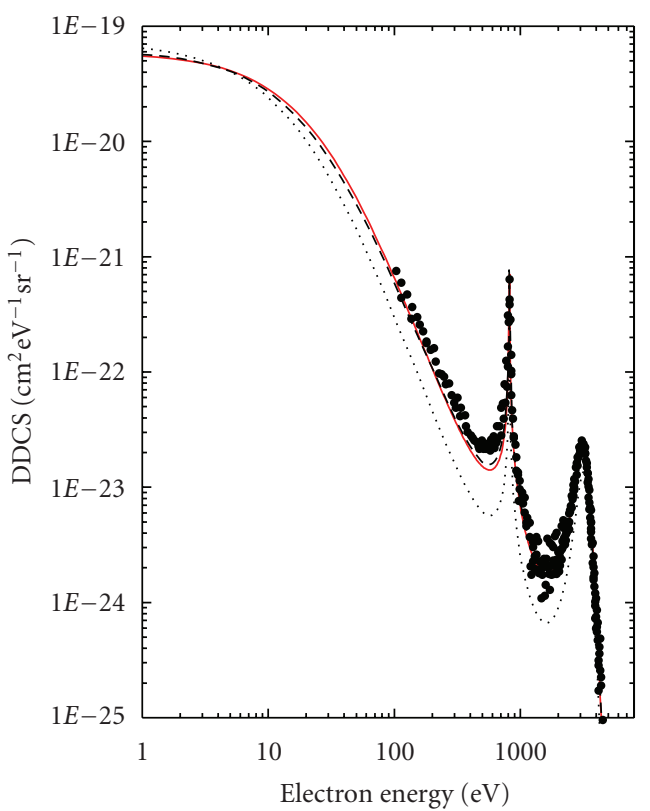

(a)

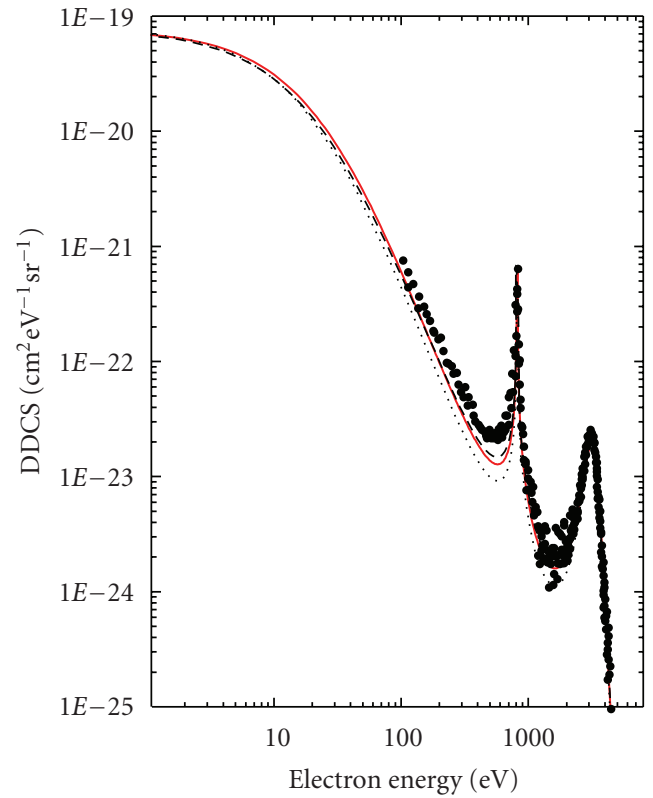

(b)

Figure 3: Doubly differential cross section for electron emission in collisions of $1.5 \mathrm{MeV} \mathrm{H}^{+}$on He targets as a function of electron energy for $0^{\circ}$ fixed ejection angle, considered in the residual target continuum function an asymptotic charge (a) or an effective charge (b). Theory: -, prior CDW-EIS; ..., post-CDW-EIS; - --, post-CDW-EIS including the terms $V_{T}^{\text {as }}$ (a) and $V_{T}^{\text {eff }}($ b). Experiment: •, Lee et al. [13].

In Section 2, a brief theoretical description is presented whereas results are discussed in Section 3, and conclusions are given in Section 4. Atomic units are used in the following except where otherwise stated.

\section{Theory}

Let us consider the single electron ionization of an atomic target of nuclear charge $Z_{T}$ in collision with a bare ion of nuclear charge $Z_{P}$.

The straight line version of the impact parameter approximation is considered, where the internuclear vector is given by the expression $\vec{R}=\vec{\rho}+\vec{v} t$, where $\vec{\rho}$ is the impact parameter and $\vec{v}$ the collision velocity, with $t$ being the evolution time taking $t=0$ at the closest distance between the nuclei.

Following Fainstein et al. [4] the multiple-body collision system can be reduced to a three-body one, so that the prior and postversions of the scattering amplitudes described from a reference frame fixed on the target nucleus can be written as

$$
\begin{aligned}
\mathcal{A}_{i f}^{-}(\vec{\rho}) & =-i \int_{-\infty}^{+\infty}\left\langle\chi_{f}^{-}\left|\left(H-\left.i \frac{\partial}{\partial t}\right|_{\vec{x}}\right)\right| \chi_{i}^{+}\right\rangle d t \\
& =-i \int_{-\infty}^{+\infty}\left\langle\chi_{f}^{-}\left|W_{i}\right| \chi_{i}^{+}\right\rangle d t, \\
\mathcal{A}_{i f}^{+}(\vec{\rho}) & =-i \int_{-\infty}^{+\infty}\left\langle\chi_{f}^{-}\left|\left(H-\left.i \frac{\partial}{\partial t}\right|_{\vec{x}}\right)^{\dagger}\right| \chi_{i}^{+}\right\rangle d t
\end{aligned}
$$

considering that the corresponding surface terms verify the conditions [9]

$$
\begin{aligned}
& \lim _{t \rightarrow+\infty}\left\langle\psi_{f}^{-} \mid \chi_{i}^{+}\right\rangle=0, \\
& \lim _{t \rightarrow-\infty}\left\langle\chi_{f}^{-} \mid \psi_{i}^{+}\right\rangle=0 .
\end{aligned}
$$

In (1) and (2), $\vec{x}$ gives the electron position with respect to the target nucleus, and $\chi_{i}^{+}$and $\chi_{f}^{-}$are the usual initial and final active electron distorted wavefunctions employed in the CDW-EIS model: $\chi_{i}^{+}=\Phi_{i}(\vec{x}, t) \mathcal{L}_{i}^{+}(\vec{s})$ and $\chi_{f}^{-}=$ $\Phi_{f}(\vec{x}, t) \mathcal{L}_{f}^{-}(\vec{s})$, where $\Phi_{i}(\vec{x}, t)$ and $\Phi_{f}(\vec{x}, t)$ represent the time-dependent target bound and continuum states of the active electron and $\vec{s}$ the electron position with respect to the projectile. $\mathcal{L}_{i}^{+}(\vec{s})$ and $\mathcal{L}_{f}^{-}(\vec{s})$ are an eikonal phase and a continuum factor, respectively, that take into account the influence of the projectile field on the active electron in the entry and exit channels, respectively. Also, $H$ is the active electron hamiltonian given by

$$
H=-\frac{1}{2} \nabla_{\vec{x}}^{2}-\frac{Z_{P}}{s}-\frac{Z_{T}}{x}+V_{\text {ap }}(\vec{x}),
$$

with $V_{\text {ap }}(\vec{x})$ being a Hartree-Fock type potential describing the influence of the passive electrons on the dynamic evolution of the active one.

The potential $W_{i}$ in (1) is the well-known EIS perturbative operator $W_{i} \chi_{i}^{+}=\Phi_{i}\left((1 / 2) \nabla_{s}^{2} \mathcal{L}_{i}^{+}(\vec{s})+\nabla_{x} \ln \varphi_{i}(\vec{x})\right.$. $\left.\nabla_{s} \mathcal{L}_{i}^{+}(\vec{s})\right)$, where $\varphi_{i}(\vec{x})$ is the time-independent initial bound wavefunction. In (3), $\psi_{i}^{+}$and $\psi_{f}^{-}$are outgoing and 


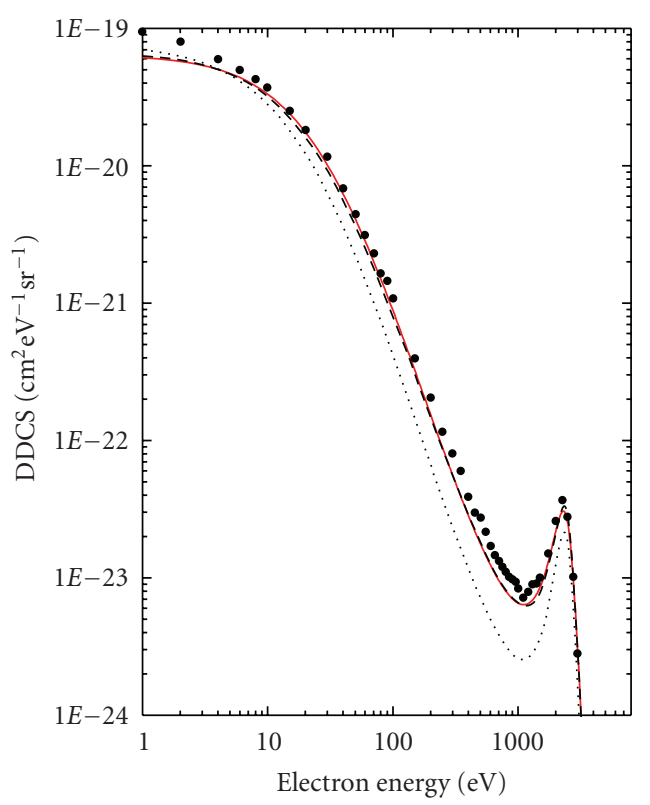

(a)

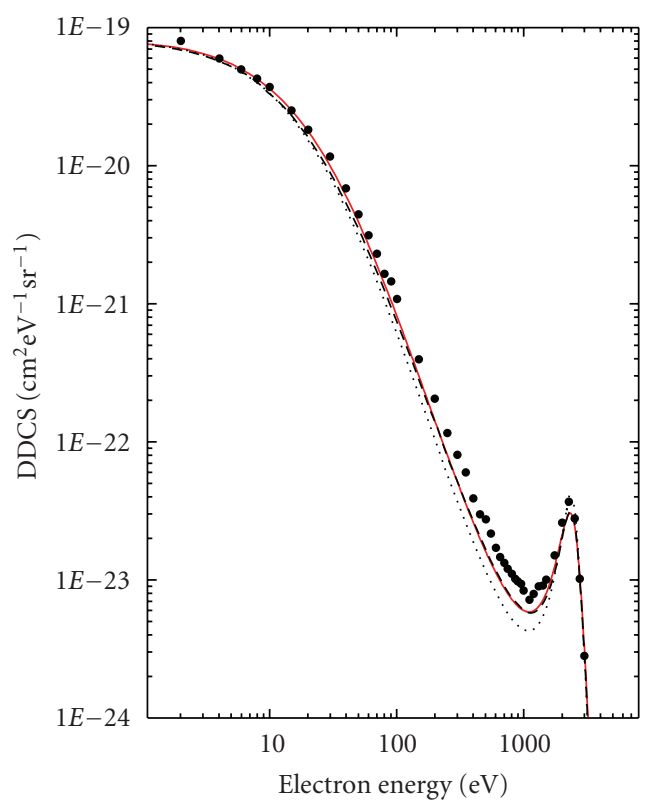

(b)

Figure 4: The same as Figure 3 but for $30^{\circ}$ fixed ejection angle. Experiment: •, Rudd et al. extracted from [12].

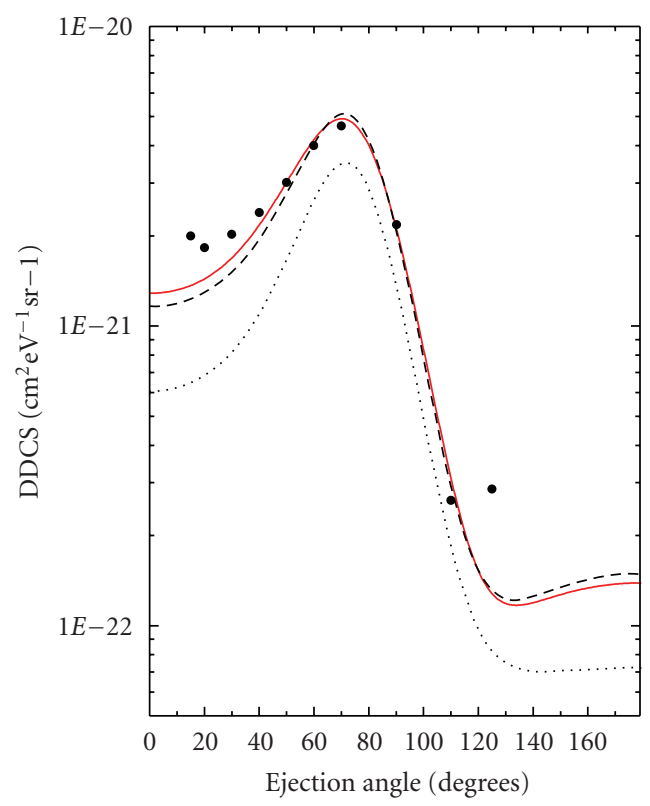

(a)

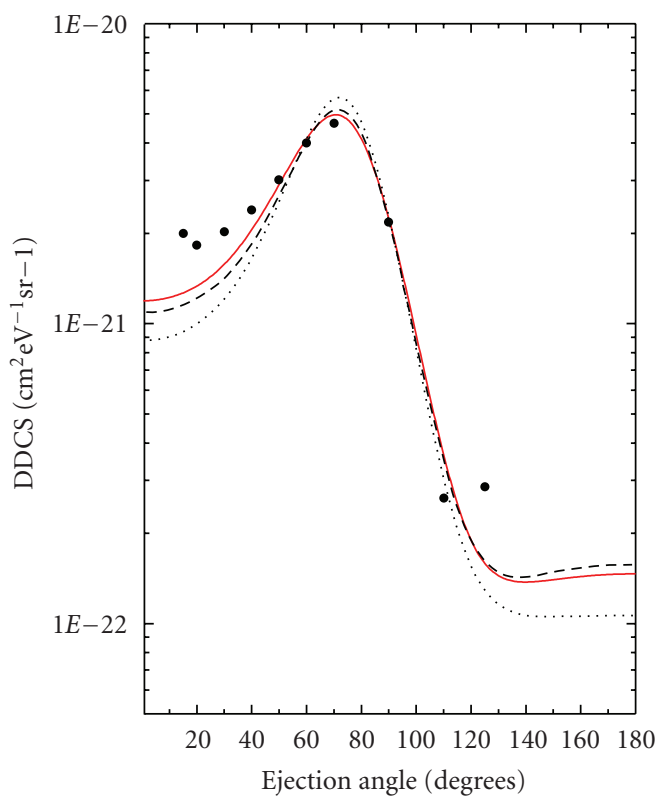

(b)

FIGURE 5: Doubly differential cross section for electron emission in collisions of $1 \mathrm{MeV} \mathrm{H}^{+}$on $\mathrm{He}$ targets as a function of ejection angle for $100 \mathrm{eV}$ fixed electron energy, considered in the residual target continuum function an asymptotic charge (a) or an effective charge (b). Theory: - , prior CDW-EIS; ..., post-CDW-EIS; - - , post-CDW-EIS including the terms $V_{T}^{\text {as }}\left(\right.$ a) and $V_{T}^{\text {eff }}($ b). Experiment: $\bullet$, extracted from Rudd et al. [12].

incoming wavefunctions that are exact solutions of the time-dependent Schrödinger equation corresponding to the hamiltonian $H$. These expressions indicate that the distorted wavefunctions do not produce by themselves the ionization transition. It must be noted that the terms involving the interactions between the nuclei and between the projectile and the passive electrons are not included in $H$ considering the well-known fact that they do not affect the calculation of cross sections integrated on the momentum transfer [4].

The potential $V_{T}(\vec{x})=-Z_{T} / x+V_{\text {ap }}(\vec{x})$ is usually approximated by an effective coulombic one: $V_{T}(\vec{x})=$ $-Z_{T}^{\text {eff }} / x$, where $Z_{T}^{\text {eff }}$ is an effective target charge chosen to 


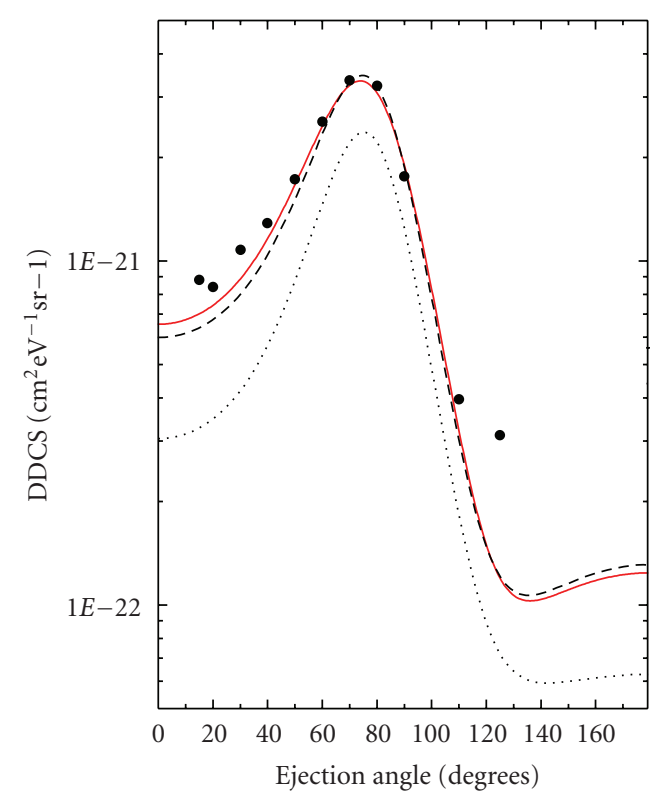

(a)

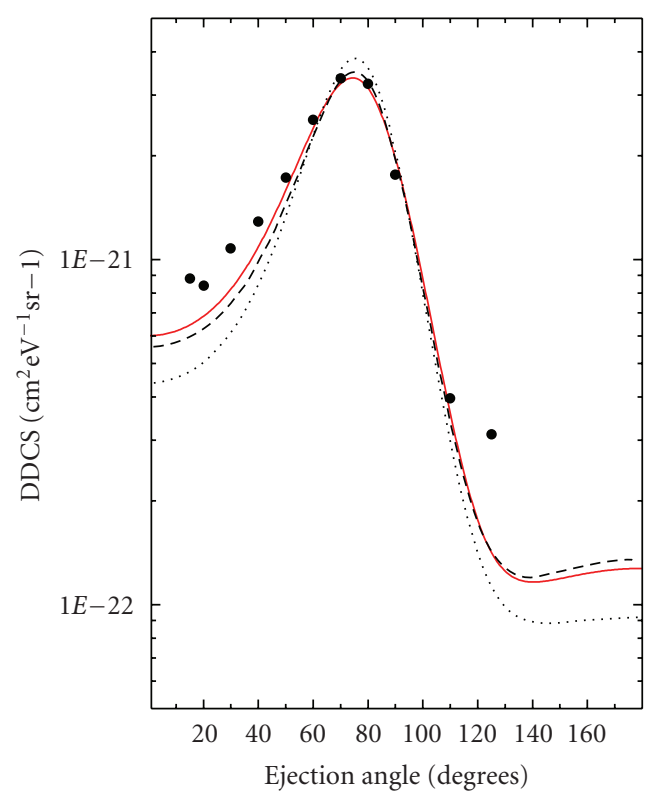

(b)

Figure 6: The same as Figure 5 but for $1.5 \mathrm{MeV}$ collision energy.

preserve the binding energy $\varepsilon_{i}$ of the active electron initial orbital. Thus, it is taken as $Z_{T}^{\text {eff }}=n_{i} \sqrt{-2 \varepsilon_{i}}$, with $n_{i}$ the principal quantum number of the orbital. With this choice, and according to Bohr's atomic picture, the region where the active electron is initially orbiting is privileged [10]. The postversion of the scattering amplitude reads, thus,

$$
\mathcal{A}_{i f}^{+}(\vec{\rho})=-i \int_{-\infty}^{+\infty}\left\langle\chi_{f}^{-}\left|W_{f}^{\dagger}+V_{T}^{\mathrm{eff}}\right| \chi_{i}^{+}\right\rangle d t,
$$

where $W_{f}^{\dagger}$ is the commonly employed CDW perturbative operator $W_{f} \chi_{f}^{-}=\Phi_{f}\left(\nabla_{x} F_{T}(\vec{x}) \cdot \nabla_{s} \mathcal{L}_{f}^{-}(\vec{s})\right)$, with $F_{T}(\vec{x})$ a residual target continuum factor [4]. The perturbative potential $V_{T}^{\text {eff }}(\vec{x})=-\left(Z_{T}-Z_{T}^{\text {eff }}\right) / x+V_{\text {ap }}$ is usually neglected in calculations. With the above given choice for $Z_{T}^{\text {eff }}$, correct boundary conditions are not satisfied in the exit channel. In order to preserve them, the target continuum wavefunction of the active electron must be chosen as the one corresponding to an asymptotic potential $-\left(Z_{T}-1\right) / x$. In fact, the ionized electron feels a charge $\left(Z_{T}-1\right)$ at large separation from the target. In such a case, the scattering amplitude becomes

$$
\mathcal{A}_{i f}^{+}(\vec{\rho})=-i \int_{-\infty}^{+\infty}\left\langle\chi_{f}^{-}\left|W_{f}^{\dagger}+V_{T}^{\mathrm{as}}\right| \chi_{i}^{+}\right\rangle d t,
$$

where now $V_{T}^{\text {as }}(\vec{x})=-\left(Z_{T}-1\right) / x+V_{\text {ap }}$. It is evident that the electron-residual target continuum factor must be selected according to the effective or asymptotic charges considered in the model. The inclusion of the perturbative potentials $V_{T}^{\text {eff }}$ and $V_{T}^{\text {as }}$ in (5) and (6), respectively, originates complete postversions of the corresponding scattering amplitudes. It allows to evaluate in first-order the role played by the dynamic screening on the evolution of the active electron in the final continuum channel. In the following, we will try to analyze its influence on doubly differential cross sections. In order to calculate them, transition matrix elements as a function of the momentum transfer are then obtained by using the well-known Fourier transform method [4]. It must be noted that in the present model, the postversion of the transition matrix element is given by an analytical expression, as it was previously found for the corresponding prior version. Finally, doubly differential cross sections are obtained by a simple integration on the momentum transfer of the square modulus of the transition matrix element [3].

\section{Results and Discussions}

The impact of protons on He targets is investigated. The initial orbital is described by a simple single-zeta function $\varphi_{i}(x)=\left(\widetilde{Z}_{T}^{3 / 2} / \pi^{1 / 2}\right) \exp \left(-\widetilde{Z}_{T} x\right)$, where $\widetilde{Z}_{T}=1.6875$ is a parameter obtained within the Roothaan-Hartree-Fock approximation [11], and the orbital energy is $\varepsilon_{i}=-0.89648$. The interaction potential between the active electron and the passive one in the exit channel is chosen as

$$
\begin{aligned}
V_{\mathrm{ap}} & =\left\langle\varphi_{i}\left(x^{\prime}\right)\left|\frac{1}{\left|\vec{x}^{\prime}-\vec{x}\right|}\right| \varphi_{i}\left(x^{\prime}\right)\right\rangle \\
& =\frac{1}{x}-\frac{\left(1+\tilde{Z}_{T} x\right)}{x} \exp \left(-2 \tilde{Z}_{T} x\right) .
\end{aligned}
$$

For the He case, $V_{T}^{\text {as }}$ results in the short-range potential

$$
V_{T}^{\text {as }}(\vec{x})=\frac{-\left(1+\tilde{Z}_{T} x\right)}{x} \exp \left(-2 \tilde{Z}_{T} x\right)
$$


which takes account of the short range part of the electron dynamic screening whereas

$$
V_{T}^{\mathrm{eff}}(\vec{x})=\frac{-\left(1-Z_{T}^{\mathrm{eff}}\right)}{x}-\frac{\left(1+\tilde{Z}_{T} x\right)}{x} \exp \left(-2 \tilde{Z}_{T} x\right)
$$

presents a coulombic asymptotic tail.

In Figures 1 and 2, double differential cross sections (DDCSs) as a function of the electron emission energy at fixed ejection angles of $\theta=0^{\circ}$ and $\theta=30^{\circ}$ are presented for impact of $1 \mathrm{MeV}$-protons. They are calculated using the prior and postversions considered in the residual target continuum function an asymptotic charge $\left(Z_{T}-1\right)$ (Figures $1(\mathrm{a})$ and $2(\mathrm{a})$ ) or an effective charge $Z_{T}^{\text {eff }}$ (Figures 1 (b) and 2(b)). The post-versions consider the inclusion or not of the corresponding perturbative potentials $V_{T}^{\text {as }}$ and $V_{T}^{\text {eff }}$. It is evidenced that post-version calculations excluding these potentials underestimate results obtained with the prior-approximation, and that including them leads to a close agreement between prior and postpredictions. In the case of using the asymptotic residual charge, the comparison between both postversions gives explicitly the influence of dynamic electron screening on the resulting DDCS. The comparison with existing experimental results shows the relevance of including such interaction. When effective charges are used, the dynamic electron screening is considered in some approximated way and so the difference between obtained DDCSs including or not the potential $V_{T}^{\text {eff }}$ in the calculations is reduced. All this analysis is also valid for results presented in Figures 3 and 4, where the case of impact of $1.5 \mathrm{MeV}$ protons was investigated.

As an example for angular distributions, in Figures 5 and 6, DDCSs as a function of the ejection angles at a fixed emission energy of $100 \mathrm{keV}$ are presented for impact of $1 \mathrm{MeV}$ and $1.5 \mathrm{MeV}$ protons, respectively. Again, for postDDCS a large underestimation of prior results is obtained when the asymptotic charge is considered. As for the energetic distributions before shown, dynamic electron screening is found to play an important role on DDCS. As it was explained for the case of effective charges, dynamic electron screening is partially included in the calculations and so the difference between post and prior calculations is reduced. However, large differences still remain with prior DDCS at small and large ejection angles when asymptotic or effective charges are considered without including the corresponding $V_{T}^{\text {as }}$ and $V_{T}^{\text {eff }}$ terms in postscattering amplitudes. In all the cases here considered, a good agreement is obtained between prior predictions and complete post ones, showing in addition a good accordance with experimental results. Differences remaining between both approximations may be attributed to the fact that in the calculations the initial bound wavefunction was described by a single-zeta RoothaanHartree-Fock representation whereas in the final channel the potential $V_{\text {ap }}$ was considered. This is independent of choosing asymptotic residual target or effective coulomb continuum wavefunctions to describe the ionized electron in the exit channel.

\section{Conclusions}

A complete three-body CDW-EIS model to describe single electron ionization is proposed, and a potential that has been excluded in previous calculations is now considered. This potential, which gives the contribution of dynamic electron screening, is shown to play a principal role in the determination of double differential cross sections. A close agreement between DDCS obtained within the prior-version and the complete post-version of the CDW-EIS model is found in the energy as well as in the angular distributions of ejected electrons. An appropriate representation of experimental data is observed. Moreover, the small post-prior discrepancies that still remain must be attributed to the different treatment employed to obtain the initial and final wavefunctions. We have recently checked that the use of more complete initial target wavefunctions does not change our main conclusions. Work along this direction is in progress. The present analysis may be extended to study ionization by multicharged ions and to investigate the case of a target with more than two electrons, assuming that the nonionized electrons remain in their initial orbitals. In order to describe fully differential cross sections, internuclear potentials and projectile-passive electron ones must be incorporated in the model. This is matter of our future research.

\section{Aknowledgments}

This work has been done as a part of investigations planned into the project PICT $\mathrm{N}^{\circ} 1912$ of the Agencia Nacional de Promoción Científica y Tecnológica of the República Argentina. The authors acknowledge this institution for financial support.

\section{References}

[1] N. Stolterfoht, R. D. DuBois, and R. D. Rivarola, Electron Emission in Heavy Ion-Atom Collisions, Springer, New York, NY, USA, 1997.

[2] Dž. Belkić, "A quantum theory of ionisation in fast collisions between ions and atomic systems," Journal of Physics B, vol. 11, no. 20, pp. 3529-3552, 1978.

[3] D. S. F. Crothers and J. F. McCann, "Ionisation of atoms by ion impact," Journal of Physics B, vol. 16, no. 17, pp. 3229-3242, 1983.

[4] P. D. Fainstein, V. H. Ponce, and R. D. Rivarola, "A theoretical model for ionisation in ion-atom collisions: application for the impact of multicharged projectiles on helium," Journal of Physics B, vol. 21, no. 2, pp. 287-299, 1988.

[5] P. D. Fainstein, V. H. Ponce, and R. D. Rivarola, "Two-centre effects in ionization by ion impact," Journal of Physics B, vol. 24, p. 3091, 1991.

[6] M. F. Ciappina, W. R. Cravero, and C. R. Garibotti, "Postprior discrepancies in the continuum distorted wave-eikonal initial state approximation for ion-helium ionization," Journal of Physics B, vol. 36, no. 18, pp. 3775-3786, 2003.

[7] M. F. Ciappina and W. R. Cravero, "Post-prior discrepancies in CDW-EIS calculations for ion impact ionization fully differential cross sections," Journal of Physics B, vol. 39, no. 5, pp. 1091-1100, 2006. 
[8] L. Gulyàs, P. D. Fainstein, and A. Salin, "CDW-EIS theory of ionization by ion impact with Hartree-Fock description of the target," Journal of Physics B, vol. 28, p. 245, 1995.

[9] A. Igarashi, L. Gulyàs, and P. D. Fainstein, "Evaluation of the single ionisation transition amplitude using the CDWEIS model within the framework of the impact-parameter method," European Physical Journal D, vol. 42, no. 1, pp. 7377, 2007.

[10] Dž. Belkić, R. Gayet, and A. Salin, "Electron capture in highenergy ion-atom collisions," Physics Reports, vol. 56, no. 6, pp. 279-369, 1979.

[11] E. Clementi and C. Roetti, "Roothaan Hartree Fock atomic wavefunctions. Basis functions and their coefficients for ground and certain excited states of neutral and ionized atoms, $\mathrm{Z} \leq 54$," Atomic Data and Nuclear Data Tables, vol. 14, no. 3-4, pp. 177-478, 1974.

[12] M. E. Rudd, L. H. Toburen, and N. Stolterfoht, "Differential cross sections for ejection of electrons from helium by protons," Atomic Data and Nuclear Data Tables, vol. 18, no. 5, pp. 413-432, 1976.

[13] D. H. Lee, P. Richard, T. J. M. Zouros, J. M. Sanders, J. L. Shinpaugh, and H. Hidmi, "Binary-encounter electrons observed at $0^{\circ}$ in collisions of $12-\mathrm{MeV} / \mathrm{amu} \mathrm{H}^{+}, \mathrm{C}^{6+}, \mathrm{N}^{7+}, \mathrm{O}^{8+}$, and $\mathrm{F}^{9+}$ ions with $\mathrm{H}_{2}$ and He targets," Physical Review A, vol. 41, no. 9, pp. 4816-4823, 1990. 

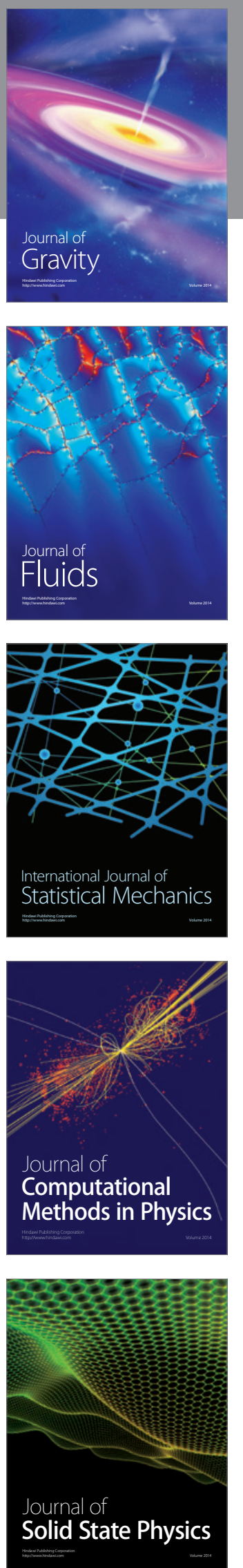

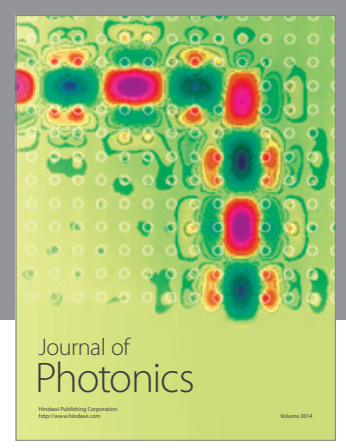

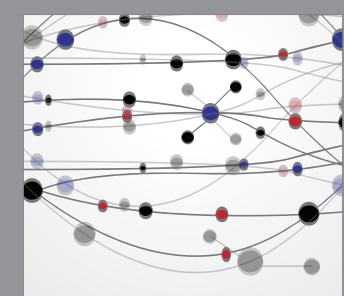

The Scientific World Journal
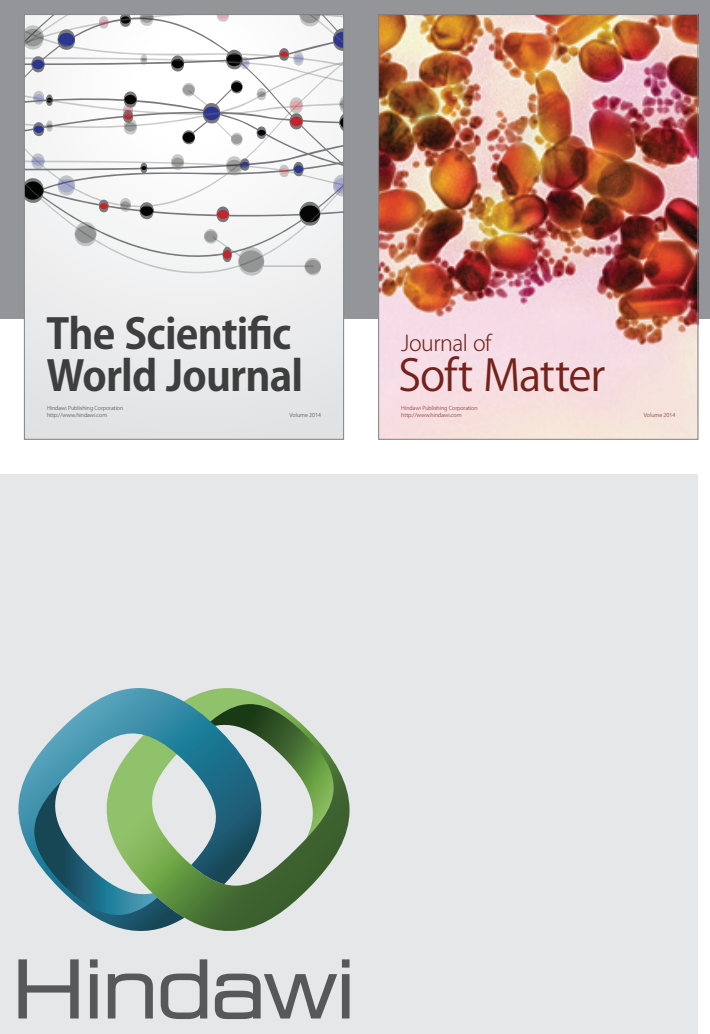

Submit your manuscripts at

http://www.hindawi.com
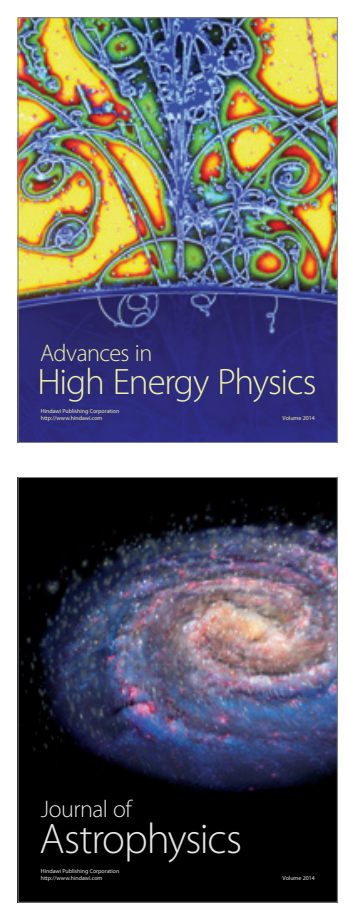
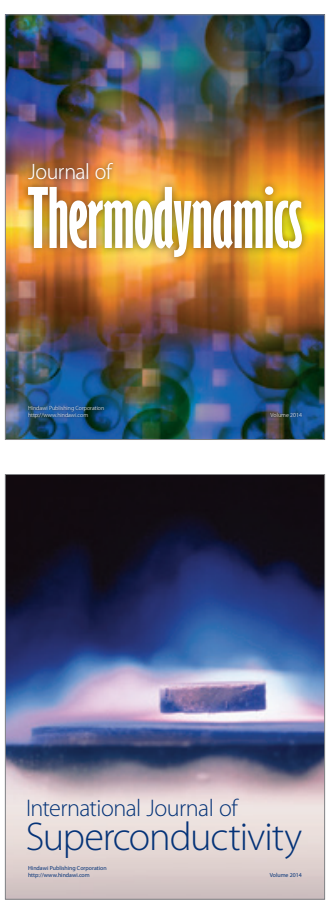
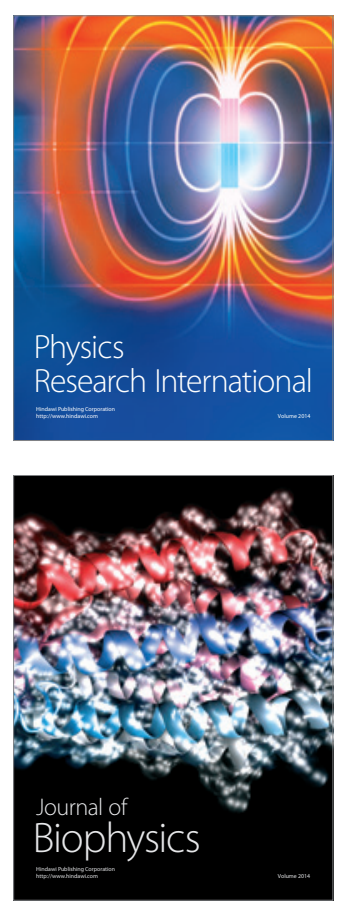
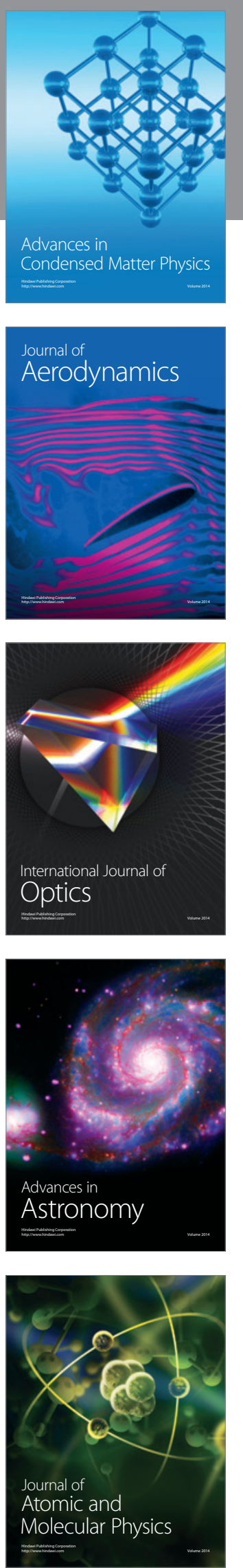